\title{
PRODUTIVIDADE E QUALIDADE DE GENÓTIPOS DE MELÃO-AMARELO EM QUATRO AMBIENTES ${ }^{1}$
}

\author{
SEBASTIÃO ELVIRO DE ARAÚJO NETO², FÁBIO DE LIMA GURGEL ${ }^{2}$, JOSUÉ FERNANDES PEDROSA², \\ REGINA LÚCIA FÉLIX FERREIRA², ANTÔNIO DE PÁDUA ARAÚJO ${ }^{4}$
}

\begin{abstract}
RESUMO - Com o objetivo de identificar genótipos de melão-amarelo, tipo exportação, com alto rendimento, foram avaliados nove genótipos em quatro ambientes no Oeste Potiguar. Os experimentos foram conduzidos no Pólo Agrícola Mossoró-Assu, Estado do Rio Grande do Norte - Brasil. O delineamento experimental foi o de blocos casualizados, com quatro repetições e 16 plantas por parcela. Os genótipos avaliados foram: Rochedo, TSX32096; PX 4910606, Gold Mine, PX-1010606; Yellow Queen; Gold Pride; Yellow King; AF-646 e AF-682. Os genótipos que apresentaram maior produtividade total, foram Rochedo, TSX-32096, Gold Mine, PX-4010606, Yellow Queen e Gold Pride, com média de 55,1 t/ha, e maior produtividade comercial foram TSX-32096, Rochedo, Gold Mine, PX-4010606 e Gold Pride em Mossoró (40,1 t/ha) e Carnaubais (46,2 t/ha), e os genótipos AF-646, Yellow Queen e AF-682 em Baraúna (63,1 t/ha), ambiente que proporcionou a maior produtividade comercial, superando em 53\%, 75\% e 166\% os ambientes de Carnaubais, Mossoró e Alto do Rodrigues. O peso médio dos frutos foi influenciado pelo ambiente de cultivo, variando de $1,07 \mathrm{~kg}$ em Mossoró a 1,64 kg em Baraúna. As maiores concentrações de SS foram observadas nos genótipos AF-646 (11,1\%), PX-4910606 (11,0\%), Yellow King $(10,7 \%)$ e TSX-32096(11,3\%).
\end{abstract}

Termos para indexação: Cucumis melo L., produção, cultivar, híbrido, melão, ambiente, genótipo.

\section{PRODUCTIVITY AND QUALITY OF GENOTYPES OF YELLOW MELON IN FOUR ENVIRONMENTS}

ABSTRACT - With the objective of identify genotypes of yellow melon export type and with high yield, were evaluated nine genotypes in four environments in Potiguar West. The experiments were established in the Mossoró-Assu Agricultural Pole, Rio Grande do Norte State - Brazil. The experimental design was in randomized blocks with four replications and 16 plants for plot. The genotypes evaluated were: Rochedo, TSX-32096; PX 4910606, Gold Mine, PX-1010606; Yellow Queen; Gold Pride; Yellow King; AF-646 and AF-682. The genotypes that presented higher total productivity were TSX-32096, Gold Mine, PX-4010606, Yellow Queen and Gold Pride, with average of 55.1 t/ha, and with higher marketable productivity were TSX32096, Rochedo, Gold Mine, PX-4010606 and Gold Pride in Mossoró (40.1 t/ha) and Carnaubais (46.2 t/ha), and the genotypes AF-646, Yellow Queen and AF-682 in Baraúna (63.1 t/ha), this environment provided the largest marketable productivity, overcoming in 53\%, $75 \%$ and $166 \%$ the environments of Carnaubais, Mossoró and Alto do Rodrigues. The fruit mean weight was influenced by the cultivation environment, ranging from $1.07 \mathrm{~kg}$ in Mossoró to $1.64 \mathrm{~kg}$ in Baraúna. The higher concentration of soluble solids was verified in the genotypes AF-646 (11.1\%), PX-4910606 (11.0\%), Yellow King (10.7\%) and TSX-32096(11.3\%).

Index terms: Cucumis melo L., yield, cultivars, hybrid, melon, environment, genotypes.

\section{INTRODUÇÃO}

A cultura do melão assume importância expressiva nos Estados da Região Nordeste, por sua posição geográfica estratégica e, principalmente, pelas condições edafoclimáticas excepcionais que, favorecendo a interação genótipo $\mathrm{x}$ ambiente, proporcionam o desenvolvimento de frutos com elevado teor de sólidos solúveis, suprindo a exigência dos países importadores. Os Estados do Rio Grande do Norte, Ceará, Paraíba, Pernambuco e Bahia é onde se concentra $90 \%$ da área total plantada no Brasil. O Rio Grande do Norte é o principal exportador, responsável por 90,6\% do melão exportado (Dias et al., 1998), produzido na região Oeste do Estado, conhecido como Pólo Agrícola Mossoró-Assu.

Apesar do excelente desempenho da cultura na região, diversos problemas de natureza técnica preocupam os produtores e demais pessoas envolvidas no processo de produção-comercialização. Dentre outros problemas que afetam a cultura, destaca-se a falta de cultivares e/ou híbridos adaptados à região em suas diversas épocas de plantio e que produzem frutos de qualidade para os comércios interno e externo.

Algumas empresas produtoras de sementes têm investido em pesquisas, visando à produção de híbridos que proporcionem rendimentos mais elevados. Como resultado dessas pesquisas, os híbridos de melão do tipo "amarelo", rapidamente, estão substituindo a cultivar Valenciano Amarelo, que, segundo Costa e Pinto (1977), é suscetível às principais doenças, que tornam a cultura da região extremamente vulnerável à ocorrência de alguma epifitotia (Lopes, 1991).

Porém, atualmente, a maioria destes híbridos utilizados tem apresentado expressiva instabilidade nos diversos ambientes de cultivo, ten- do conseqüências na produção.

A maneira usual para a solução de problemas dessa natureza tem sido, segundo Torres Filho et al. (1987), o teste de capacidade produtiva de certo número de genótipos, cultivados por vários anos e locais, procedendo-se assim à análise conjunta desses genótipos e comparando-as em relação à média da população.

Assim, este trabalho tem como objetivo identificar genótipos de melão-amarelo com altos rendimentos para os ecossistemas do Pólo Agrícola Mossoró-Assu.

\section{MATERIAL EMÉTODOS}

A pesquisa foi desenvolvida na mesorregião Oeste do Estado do Rio Grande do Norte, onde se localiza o Pólo Agrícola Mossoró-Assu, responsável por praticamente toda a produção de melão do Estado. Foram selecionadas áreas de cultivo comercial em quatro municípios: Mossoró, Carnaubais, Alto do Rodrigues e Baraúna, nos quais foram instalados os experimentos durante o ano agrícola de 1998-1999.

A localização dos municípios é: Mossoró $5^{\circ} 11^{\prime} \mathrm{S}$ e $37^{\circ} 21^{\prime} \mathrm{O}$; Alto do Rodrigues $5^{\circ} 16^{\prime} \mathrm{S}$ e $36^{\circ} 12^{\prime} \mathrm{O}$; Carnaubais 5 $5^{\circ} 20^{\prime} \mathrm{S}$ e $36^{\circ} 50^{\prime} \mathrm{O}$; Baraúna $5^{\circ} 05^{\prime} \mathrm{Se} 37^{\circ} 38^{\prime} \mathrm{O}$.

As condições de fertilidade do solo e a qualidade da água dos locais onde foram instalados os experimentos estão contidas nas Tabelas 1 e 2 , respectivamente.

O delineamento experimental utilizado foi o de blocos casualizados completos, com nove tratamentos e quatro repetições. Os tratamentos consistiram de nove genótipos oriundos de empresas produ-

\footnotetext{
${ }^{1}$ (Trabalho 074/2002). Recebido: 26/04/2002. Aceito para publicação: 05/03/2003.

${ }^{2}$ Eng $^{\circ}$. Agro ${ }^{\circ}$ M.Sc. Fitotecnia/ESAM, Doutorandos da Universidade Federal de Lavras-UFLA. Rua Francisco Xavier, 414, Vila São Francisco, Lavras-MG. e-mail: selviro@zipmail.com.br; flgurgel@msn.com; reginalff@hotmail.com.

${ }^{3}$ Prof. Adjunto da Universidade Estadual do Rio Grande do Norte - UERN. Fone: (0xx84) 314-2072.

${ }^{4}$ Eng $^{\circ}$. Agr ${ }^{\circ}$. M.Sc. Fitotecnia/ESAM. Rua Eufrasina Fernandes, 70, Assu-RN, e-mail: araujoap@zipmail.com.br.
} 
toras de sementes (Rochedo, TSX-32096; PX 4910606, Gold Mine, PX1010606; Yellow Queen; Gold Pride; Yellow King; AF-646 eAF-682). Cada parcela ficou constituída por duas linhas de plantio de 5,0 metros de comprimento, contendo 10 covas com 2 plantas cada linha, espaçadas $2,0 \times 0,5$ $\mathrm{m}$, obedecendo ao mesmo espaçamento dos gotejadores. Foi considerada área útil o conjunto de 16 plantas centrais de uma das linhas. O experimento ficou, portanto, com uma densidade populacional de 20.000 plantas/ha.

TABELA 1- Caracterização da fertilidade dos solos da área dos experimentos.

\begin{tabular}{|c|c|c|c|c|c|c|c|c|c|c|}
\hline \multirow[t]{2}{*}{ Locais } & \multirow{2}{*}{$\begin{array}{c}\mathbf{p H} \\
\mathrm{H}_{2} \mathrm{O}\end{array}$} & \multirow{2}{*}{$\begin{array}{c}\text { P(resina) } \\
\mu \text { g.cm }\end{array}$} & $\mathbf{S}$ & $\mathrm{Ca}$ & Mg & $\mathbf{K}$ & $\mathrm{Na}$ & $\mathbf{A L}$ & CTC & \multirow{2}{*}{$\begin{array}{r} \\
(\%)\end{array}$} \\
\hline & & & \multicolumn{7}{|c|}{$\mathrm{cmol}_{\mathrm{c}} \cdot \mathrm{dm}^{-3}$} & \\
\hline Mossoró & 8,3 & 6,1 & - & 6,50 & 4,50 & 2,18 & 1,70 & 0,00 & - & - \\
\hline Carnaubais & 6,8 & 29,0 & 68 & 1,70 & 0,44 & 0,34 & 0,05 & 0,00 & 2,53 & 100 \\
\hline Alto do Rodrigues & 7,4 & 6,0 & 23 & 16,80 & 1,48 & 0,42 & 0,09 & 0,00 & 17,90 & 98 \\
\hline \multirow[t]{3}{*}{ Baraúna } & 6,5 & 2,0 & 05 & 4,25 & 1,03 & 0,25 & 0,05 & 1,29 & 6,87 & 81 \\
\hline & \multicolumn{2}{|r|}{$\mathrm{Cu}$} & \multicolumn{2}{|c|}{ Zn } & \multicolumn{2}{|c|}{$\mathrm{Fe}$} & \multicolumn{2}{|c|}{ Mn } & \multicolumn{2}{|c|}{ B } \\
\hline & \multicolumn{10}{|c|}{$\mathrm{mg.dm}^{-3}$} \\
\hline Mossoró & \multicolumn{2}{|r|}{-} & \multicolumn{2}{|r|}{-} & \multicolumn{2}{|c|}{-} & \multicolumn{2}{|c|}{-} & \multicolumn{2}{|c|}{-} \\
\hline Carnaubais & \multicolumn{2}{|r|}{0,24} & \multicolumn{2}{|c|}{1,0} & \multicolumn{2}{|c|}{11} & \multicolumn{2}{|c|}{58,5} & \multicolumn{2}{|c|}{0,7} \\
\hline Alto do Rodrigues & \multicolumn{2}{|r|}{0,11} & \multicolumn{2}{|c|}{0,4} & \multicolumn{2}{|c|}{06} & \multicolumn{2}{|c|}{54,8} & \multicolumn{2}{|c|}{0,9} \\
\hline Baraúna & \multicolumn{2}{|r|}{1,94} & \multicolumn{2}{|c|}{1,0} & \multicolumn{2}{|c|}{18} & \multicolumn{2}{|c|}{69,3} & \multicolumn{2}{|c|}{0,6} \\
\hline
\end{tabular}

TABELA 2- Caracterização e classificação da água disponível para irrigação nos locais em que foram instalados os experimentos.

\begin{tabular}{|c|c|c|c|c|c|c|c|c|c|c|c|c|}
\hline Locais & $\mathrm{pH}$ & $\begin{array}{c}\mathrm{CE} \\
\left(\mathrm{ms}^{\left.-\mathrm{cm}^{-1}\right)}\right.\end{array}$ & $\underset{\left(\mathrm{mg} . \mathrm{I}^{-1}\right)}{\mathrm{SD}}$ & RAS & $\mathrm{Ca}$ & Mg & K & $\frac{\mathrm{Na}}{\mathrm{meq} / \mathrm{l}}$ & $\mathrm{CO}_{3}$ & $\mathrm{HCO}_{3}$ & $\mathrm{Cl}$ & $\begin{array}{c}\text { Classificação } \\
\text { da água }\end{array}$ \\
\hline Mossoró & 8,1 & 0,55 & - & 2,32 & 2,00 & 0,90 & 0,40 & 2,90 & 0,20 & 4,00 & 1,80 & $\mathrm{C} 2-\mathrm{S} 1$ \\
\hline Carnaubais & 8,4 & 0,29 & 185,6 & 2,00 & 0,72 & 0,66 & 0,20 & 1,25 & 0,09 & 1,65 & 1,32 & $\mathrm{C} 2-\mathrm{S} 1$ \\
\hline Alto do Rodrigues & 7,9 & 0,33 & 209,3 & 1,50 & 0,85 & 0,70 & 0,21 & 1,29 & 0,17 & 1,78 & 1,36 & $\mathrm{C} 2-\mathrm{S} 1$ \\
\hline Baraúna & 7,1 & 1,18 & 753,3 & 1,11 & 7,02 & 2,62 & 0,06 & 2,44 & 0,00 & 7,74 & 4,00 & C3-S1 \\
\hline
\end{tabular}

O preparo do solo da área experimental (aração, gradagem, sulcamento e fechamento) para implantação da cultura, bem como a instalação e correção do sistema de irrigação, foi realizado de acordo com recomendações para a cultura.

A cultura foi irrigada pelo método de gotejamento, com fertirrigação, utilizando o programa de adubação recomendado para a cultura, após avaliação da fertilidade do solo. O replantio foi realizado uma semana após o plantio. O controle das plantas daninhas foi realizado através de capinas manuais. As pulverizações com fungicidas foram realizadas em função do nível de infestação, e, a partir do décimo dia da implantação, as aplicações ocorreram semanalmente. A viragem dos frutos ocorreu entre 8 e 10 dias antes da colheita. Os frutos foram colhidos no estádio de maturação comercial.

As características avaliadas foram: produtividade total, através da pesagem de todos os frutos da área útil da parcela de cada tratamento, sendo estimado para $\mathrm{t} / \mathrm{ha}$; produtividade comercial, pesando apenas os frutos com qualidade comercial (frutos com ausência de danos e mancha na casca) expresso em $\mathrm{t} / \mathrm{ha}$; peso médio dos frutos, expresso em $\mathrm{kg} /$ fruto e sólidos solúveis (SS). A concentração de sólidos solúveis foi determinada através de refratômetro digital modelo PR-100 Palette, com correção automática de temperatura (escala de 0 a $32 \%$ ), pela retirada de uma fatia longitudinal de cada um dos três frutos que compõem cada parcela experimental. Em seguida, procedeu-se à homogeneização da polpa destas três fatias em um liquidificador doméstico. O suco obtido após o processamento foi filtrado em papel de filtro. Foram retiradas algumas gotas do filtrado com uma pipeta, para a realização de três leituras, através das quais se obteve o valor médio da parcela, expresso em porcentagem (\%).

Estas características foram submetidas à análise de variância de cada experimento, separadamente, seguindo as recomendações de Gomes (1990). Em seguida, procedeu-se à análise conjunta dos experimentos, de acordo com as sugestões de Ferreira (2000).

\section{RESULTADOS E DISCUSSÃO}

O resultado da análise conjunta da produtividade total e produtividade comercial revelou efeito do genótipo $(\mathrm{G})$, do ambiente $(\mathrm{A})$ e da interação (G x A). Para a característica Peso médio de fruto, houve efeito apenas do genótipo e do ambiente isolados. Para SS, houve efeito do genótipo e do ambiente para todos os experimentos, mas foi possível verificar significância da interação, apenas entre os ambientes de Mossoró e Carnaubais, por terem o $\mathrm{QM}_{\text {residuo }}$ homogêneo, e com isso foi possível realizar apenas o teste de média entre os genótipos dentro dos ambientes Alto do Rodrigues e Baraúna.

A produtividade total variou de 47,0 a $59,4 \mathrm{t} / \mathrm{ha}$, maior que a média da região $(47,7 \mathrm{t} / \mathrm{ha})$, com maior rendimento para os genótipos Rochedo, TSX-32096, Gold Mine, PX-4010606, Yellow Queen e Gold Pride, que não diferiram entre si, com média de 55,1 t/ha. Com relação aos ambientes, esses genótipos também apresentaram maiores produtividades, com exceção em Alto do Rodrigues, em que os genótipos TSX-32096, Gold Mine e Gold Pride e em Baraúna o PX-4910606, não foram os mais produtivos (Tabela 3). O mesmo desempenho foi observado para a produtividade comercial dos genótipos TSX-32096, Rochedo, Gold Mine, PX-4010606 e Gold Pride em Mossoró (40,1 t/ha) e Carnaubais (46,2 t/ha), incluindo os genótipos AF-646, Yellow Queen e AF-682 em Baraúna (63,1 $\mathrm{t} / \mathrm{ha}$ ), ambiente que proporcionou a maior produtividade comercial, superando em 53\%, 75\% e 166\% os ambientes de Carnaubais, Mossoró e Alto do Rodrigues, respectivamente (Tabela 4).

TABELA 3- Produtividade total ( $\mathrm{t} / \mathrm{ha}$ ) para os genótipos de melão-amarelo, cultivados em quatro ambientes. Mossoró - RN, 2000.

\begin{tabular}{|c|c|c|c|c|c|}
\hline \multirow[b]{2}{*}{ Genótipos } & \multicolumn{4}{|c|}{ Ambientes } & \multirow{2}{*}{$\begin{array}{l}\text { Média dos } \\
\text { genótipos }\end{array}$} \\
\hline & Mossoró & Carnaubais & $\begin{array}{c}\text { Alto do } \\
\text { Rodrigues }\end{array}$ & Baraúna & \\
\hline Rochedo & $49,3 \mathrm{a}^{*}$ & $61,9 \mathrm{a}$ & $63,2 a b c$ & $63,3 \mathrm{ab}$ & 59,4 a \\
\hline TSX-32096 & $46,0 \mathrm{a}$ & $61,3 \mathrm{a}$ & $44,1 \mathrm{~d}$ & $74,9 \mathrm{a}$ & $56,6 \mathrm{a}$ \\
\hline Gold Mine & $50,1 \mathrm{a}$ & $53,6 \mathrm{a}$ & 49,6 bcd & $72,9 \mathrm{a}$ & $56,5 \mathrm{ab}$ \\
\hline PX-4010606 & $47,6 \mathrm{a}$ & 59,4 a & $62,1 \mathrm{abcd}$ & $51,8 \mathrm{~b}$ & $55,2 \mathrm{abc}$ \\
\hline Yellow & $42,7 \mathrm{ab}$ & $43,5 \mathrm{a}$ & $69,5 \mathrm{a}$ & $61,6 \mathrm{ab}$ & $54,3 \mathrm{abc}$ \\
\hline \multicolumn{6}{|l|}{ Queen } \\
\hline Gold Pride & $47,2 \mathrm{a}$ & $52,4 \mathrm{a}$ & $46,7 \mathrm{~cd}$ & $59,6 \mathrm{ab}$ & $51,5 \mathrm{abc}$ \\
\hline Yellow King & $39,4 \mathrm{ab}$ & $39,7 \mathrm{a}$ & $67,6 \mathrm{ab}$ & $53,1 \mathrm{~b}$ & 49,9 bc \\
\hline AF-646 & $27,5 \mathrm{ab}$ & $53,6 \mathrm{a}$ & 49,2 bcd & $66,9 \mathrm{ab}$ & $49,3 \mathrm{bc}$ \\
\hline AF-682 & $18,9 \mathrm{c}$ & $48,4 \mathrm{a}$ & $56,1 \mathrm{abcd}$ & $64,7 \mathrm{ab}$ & $47,0 \mathrm{c}$ \\
\hline $\begin{array}{l}\text { Média do } \\
\text { ambiente }\end{array}$ & $41,0 \mathrm{C}$ & $52,7 \mathrm{~B}$ & $56,5 \mathrm{~B}$ & $63,2 \mathrm{~A}$ & \\
\hline C.V. $(\%)$ & 24,6 & 14,6 & 24,2 & 14,1 & \\
\hline
\end{tabular}

* Médias seguidas pela mesma letra minúscula nas colunas e maiúscula na linha não diferem significativamente, pelo teste Duncan, a $5 \%$ de probabilidade. 
TABELA 4- Produtividade comercial (t/ha) para os genótipos de melãoamarelo, cultivados em quatro ambientes. Mossoró - RN, 2000.

\begin{tabular}{|c|c|c|c|c|c|}
\hline \multirow{2}{*}{ Genótipos } & \multicolumn{4}{|c|}{ Ambientes } & \multirow{2}{*}{$\begin{array}{l}\text { Média dos } \\
\text { genótipos }\end{array}$} \\
\hline & Mossoró & Carnaubais & $\begin{array}{c}\text { Alto do } \\
\text { Rodrigues }\end{array}$ & Baraúna & \\
\hline TSX-32096 & $36,6 \mathrm{a}^{*}$ & $52,7 \mathrm{a}$ & $22,7 \mathrm{ab}$ & $74,9 \mathrm{a}$ & $46,7 \mathrm{a}$ \\
\hline Rochedo & $41,4 \mathrm{a}$ & $51,1 \mathrm{a}$ & $32,3 \mathrm{a}$ & $60,4 a b c$ & $46,3 \mathrm{a}$ \\
\hline Gold Mine & $42,7 \mathrm{a}$ & $43,3 \mathrm{ab}$ & $19,9 \mathrm{ab}$ & $67,2 \mathrm{ab}$ & $43,3 \mathrm{ab}$ \\
\hline PX-4010606 & $40,7 \mathrm{a}$ & $45,2 \mathrm{ab}$ & $31,8 \mathrm{a}$ & $48,1 \mathrm{c}$ & $41,5 \mathrm{abc}$ \\
\hline Gold Pride & 38,9 a & $42,3 \mathrm{ab}$ & $17,4 \mathrm{ab}$ & $55,9 \mathrm{bc}$ & 38,6 bcd \\
\hline AF-646 & $22,7 \mathrm{bc}$ & $33,3 \mathrm{bc}$ & $19,7 \mathrm{ab}$ & $64,8 \mathrm{ab}$ & $35,1 \mathrm{~cd}$ \\
\hline Yellow Queen & $38,7 \mathrm{a}$ & $30,4 \mathrm{bc}$ & $12,1 \mathrm{~b}$ & $60,8 \mathrm{abc}$ & $35,5 \mathrm{~cd}$ \\
\hline Yellow King & $34,3 \mathrm{ab}$ & $26,3 \mathrm{c}$ & $30,9 \mathrm{a}$ & $48,9 \mathrm{c}$ & $35,1 \mathrm{~cd}$ \\
\hline AF-682 & $15,2 \mathrm{c}$ & $33,0 \mathrm{bc}$ & $17,7 \mathrm{ab}$ & $63,7 \mathrm{ab}$ & $32,4 \mathrm{~d}$ \\
\hline $\begin{array}{l}\text { Média do } \\
\text { ambiente }\end{array}$ & $34,6 \mathrm{C}$ & $39,7 \mathrm{~B}$ & $22,7 \mathrm{D}$ & $60,5 \mathrm{~A}$ & \\
\hline C.V. (\%) & 27,4 & 20,4 & 29,2 & 15,8 & \\
\hline
\end{tabular}

${ }^{*}$ Médias seguidas pela mesma letra minúscula nas colunas e maiúscula na linha não diferem significativamente, pelo teste Duncan, a 5\% de probabilidade.

Os relatos indicam que esses genótipos se adaptam muito bem às condições do Nordeste brasileiro, apresentando alta produtividade total, com média de 47,7 t/ha, variando pouco entre si, verificado em trabalhos de avaliação de cultivares (Tabela 5). Mas o ambiente influencia muito o desempenho dos genótipos, o que aconteceu com o aumento de produtividade total e comercial dos genótipos AF-646, Yellow Queen e AF-682 em Baraúna, em relação a Mossoró e Carnaubais, a mesma observação em Juazeiro-BA, em que esses genótipos superaram os demais na avaliação feita por Costa et al. (2000).

TABELA 5- Índices de produtividade, peso médio do fruto e concentração de sólidos solúveis para genótipos de melão-amarelo, cultivados no Nordeste brasileiro.

\begin{tabular}{|c|c|c|c|c|c|}
\hline \multirow[t]{2}{*}{ Genótipos } & \multicolumn{2}{|c|}{ Produtividade } & \multirow{2}{*}{$\begin{array}{l}\text { Peso Médio } \\
\text { (kg/fruto) }\end{array}$} & \multirow{2}{*}{$\begin{array}{l}\text { SS } \\
(\%)\end{array}$} & \multirow[t]{2}{*}{ Autores } \\
\hline & $\begin{array}{l}\text { Total } \\
(\mathrm{t} / \mathrm{ha})\end{array}$ & $\begin{array}{c}\text { Comercial } \\
(\mathrm{t} / \mathrm{ha})\end{array}$ & & & \\
\hline Yellow Queen & 50,70 & 39,20 & 2,02 & 10,61 & 2,5 \\
\hline Gold Mine & 48,80 & 38,33 & 1,78 & 10,60 & $1,2,3,4,5,6,7,8$ \\
\hline Rochedo & 48,25 & 39,20 & 1,96 & 9,67 & $2,5,8$ \\
\hline AF-682 & 48,73 & 46,53 & 1,80 & 10,97 & $2,4,5,8$ \\
\hline AF-646 & 46,41 & 34,30 & 1,54 & 11,44 & $2,4,5,7,8$ \\
\hline Yellow King & 45,87 & 35,33 & 1,75 & 11,09 & 2,5 \\
\hline Gold Pride & 45,40 & 37,37 & 1,59 & 10,57 & $2,4,5,8$ \\
\hline Média & 47,74 & 38,61 & 1,78 & 10,71 & \\
\hline Desvio Padrão & 1,91 & 3,97 & 0,18 & 0,56 & \\
\hline
\end{tabular}

Autores: 1-Araujo et al., 2000; 2-Costa et al., 2000; 3-Silva et al., 2000a; 4-Silva et al., 2000b; 5-Nunes et al., 2000; 6-Pinheiro Neto et al., 2001; 7-Silva et al., 2001; 8-Costa et al., 2001

Do melão produzido em Baraúna, 95,7\% foi classificado como comercial, enquanto em Alto do Rodrigues somente 40,2\% do melão produzido se enquadrou nos padrões de comercialização; a razão por essa baixa produtividade comercial deveu-se à ocorrência de doenças, saúvas e ventos fortes na região, características que não foram preestabelecidas no projeto, e por isso não foram analisadas e caracterizadas, não evidenciado neste trabalho por não ter sido predeterminado como metodologia.

O peso médio dos frutos contribui com o rendimento, como ocorreu com o genótipo Rochedo, que produziu um número total de frutos menor que o PX-4010606 e Gold Pride (dados não apresentados) e compensado com o maior peso médio do fruto, de 1,53 kg (Tabela 6), constitui, também, importante parâmetro de comercialização.

Os genótipos Rochedo, Yellow Queen, Gold Mine, AF-682, Yellow King, PX-4910606 e TSX-32096 não diferiram entre si, e os menores pesos médios foram observados para Gold Pride e AF-646. Mas, em geral, produziram frutos com peso médio abaixo da média da região Nordeste $(1,78 \mathrm{~kg})$, fato desejável para o mercado exportador, que prefere frutos menores, ficando os maiores para o mercado interno.

TABELA 6-Peso médio do fruto $(\mathrm{kg})$ para os genótipos de melão-amarelo, cultivados em quatro ambientes. Mossoró - RN, 2000.

\begin{tabular}{|c|c|c|c|c|c|}
\hline \multirow[b]{2}{*}{ Genótipos } & \multicolumn{4}{|c|}{ Ambientes } & \multirow{2}{*}{$\begin{array}{c}\text { Média } \\
\text { dos } \\
\text { genótipos }\end{array}$} \\
\hline & Mossoró & Carnaubais & $\begin{array}{c}\text { Alto do } \\
\text { Rodrigues }\end{array}$ & Baraúna & \\
\hline Rochedo & 1,10 & 1,68 & 1,59 & 1,75 & $1,53 \mathrm{a}^{4}$ \\
\hline Yellow Queen & 1,26 & 1,58 & 1,35 & 1,74 & $1,48 \mathrm{ab}$ \\
\hline Gold Mine & 1,09 & 1,72 & 1,25 & 1,78 & $1,45 \mathrm{ab}$ \\
\hline AF-682 & 1,24 & 1,58 & 1,40 & 1,55 & $1,44 \mathrm{ab}$ \\
\hline Yellow King & 1,11 & 1,44 & 1,43 & 1,67 & $1,41 \mathrm{ab}$ \\
\hline PX-4010606 & 1,01 & 1,60 & 1,26 & 1,51 & $1,34 \mathrm{ab}$ \\
\hline TSX-32096 & 1,01 & 1,58 & 1,29 & 1,48 & $1,34 \mathrm{ab}$ \\
\hline Gold Pride & 0,92 & 1,43 & 1,16 & 1,72 & $1,31 \mathrm{~b}$ \\
\hline AF-646 & 0,95 & 1,39 & 1,03 & 1,55 & $1,23 \mathrm{c}$ \\
\hline Média do & $1,07 \mathrm{C}$ & $1,56 \mathrm{~A}$ & $1,31 \mathrm{~B}$ & $64 \mathrm{~A}$ & \\
\hline
\end{tabular}

\begin{tabular}{lrrrr}
$\begin{array}{l}\text { Media do } \\
\text { ambiente }\end{array}$ & $1,07 \mathrm{C}$ & $1,56 \mathrm{~A}$ & $1,31 \mathrm{~B}$ & $1,64 \mathrm{~A}$ \\
\hline C.V. $(\%)$ & 16,0 & 11,2 & 20,2 & 8,6 \\
\hline
\end{tabular}

${ }^{*}$ Médias seguidas pela mesma letra minúscula nas colunas e maiúscula na linha não diferem significativamente, pelo teste Duncan, a 5\% de probabilidade.

O peso médio dos frutos foi influenciado pelo ambiente de cultivo, variando de 1,07 kg em Mossoró, a 1,64 kg em Baraúna, contribuindo para obter-se maiores rendimentos neste último.

O teor de sólidos solúveis para a maioria dos genótipos cultivados nos municípios de Mossoró, Carnaubais e alguns do Alto do Rodrigues não se enquadra na escala de melões aptos à comercialização, pela metodologia proposta por Gorgatti Netto et al. (1994), que sugere a obtenção dos teores de SS, utilizando-se apenas do suco extraído da polpa localizada na região equatorial do fruto, onde há maior concentração de açúcares (Scott \& MacGillvray, 1940). Desta forma, não há uma precisão nos reais valores do teor de SS existentes nos frutos dos genótipos avaliados (Tabela 7).

TABELA 7-Sólidos solúveis (\%) de genótipos de melão-amarelo, cultivados em quatro ambientes. Mossoró - RN, 2000.

\begin{tabular}{|c|c|c|c|c|c|}
\hline \multirow{2}{*}{ Genótipos } & \multicolumn{2}{|c|}{ Ambientes } & \multirow{2}{*}{$\begin{array}{c}\text { Média dos } \\
\text { genótipos } \\
\text { de M e C }\end{array}$} & \multirow{2}{*}{$\begin{array}{c}\text { Alto do } \\
\text { Rodrigues }\end{array}$} & \multirow[t]{2}{*}{ Baraúna } \\
\hline & Mossoró & Carnaubais & & & \\
\hline TSX-320960 & $8,68 \mathrm{a}^{*}$ & $9,70 \mathrm{a}$ & $9,19 \mathrm{a}^{*}$ & $10,23 \mathrm{a}$ & $11,28 \mathrm{a}$ \\
\hline Yellow King & $7,83 \mathrm{~b}$ & $7,85 \mathrm{~b}$ & $7,84 \mathrm{~b}$ & $7,60 \mathrm{~h}$ & $10,70 \mathrm{~d}$ \\
\hline AF-646 & $7,15 \mathrm{~b}$ & $8,25 \mathrm{bc}$ & $7,70 \mathrm{~b}$ & $8,33 \mathrm{~g}$ & $11,10 \mathrm{~b}$ \\
\hline Gold Mine & $7,53 \mathrm{~b}$ & $7,68 \mathrm{bc}$ & $7,60 \mathrm{bc}$ & $9,08 \mathrm{e}$ & $9,93 \mathrm{f}$ \\
\hline Rochedo & $7,75 \mathrm{~b}$ & $7,58 \mathrm{bc}$ & $7,66 \mathrm{bc}$ & $9,63 \mathrm{c}$ & $9,80 \mathrm{~g}$ \\
\hline Gold Pride & $7,43 \mathrm{~b}$ & $7,48 \mathrm{bc}$ & $7,45 \mathrm{bc}$ & $9,58 \mathrm{c}$ & $8,13 \mathrm{i}$ \\
\hline Yellow Queen & $7,53 \mathrm{~b}$ & $7,23 \mathrm{c}$ & $7,34 \mathrm{bc}$ & $9,23 \mathrm{~d}$ & $10,23 \mathrm{e}$ \\
\hline PX-4010606 & $6,25 \mathrm{c}$ & $7,82 \mathrm{bc}$ & $7,04 \mathrm{c}$ & $8,70 \mathrm{f}$ & $11,00 \mathrm{c}$ \\
\hline AF-682 & $6,30 \mathrm{c}$ & $7,78 \mathrm{bc}$ & $7,04 \mathrm{c}$ & $9,91 \mathrm{~b}$ & $9,33 \mathrm{~h}$ \\
\hline Média do & 7,38 B & 7,93 A & & 9,14 & 10,16 \\
\hline
\end{tabular}

ambiente

\begin{tabular}{lllll}
\hline C.V. $(\%)$ & 6,3 & 7,4 & 1,1 & 0,6
\end{tabular}

" Médias seguidas pela mesma letra minúscula nas colunas e maiúscula na linha não diferem significativamente, pelo teste Duncan, a $5 \%$ de probabilidade.

${ }^{* *}$ Médias dos genótipos dos ambientes Mossoró e Carnaubais, por terem QM homogêneo.

Assim, há uma diferença para mais duas unidades $\left(2^{\circ}\right.$ Brix $)$ entre os dados analisados, para que se possa classificar os frutos pela escala daquele autor. Procedendo-se desta forma, todos os ambientes proporcionaram frutos aptos à comercialização, dentre os quais Alto do Rodrigues e Baraúna apresentaram os maiores índices.

Os genótipos que se destacaram, foram o TSX-32096 (11,3\%), AF-646(11,1\%), PX-4910606(11,0\%) e Yellow King (10,7\%), sendo considerados melões extras, principalmente quando cultivados em Baraúna. Esses valores estão semelhantes aos encontrados no genótipo TSX-32096 $(12,4 \%)$, avaliado por Menezes et al. (2001), portanto, acima do exigido pelos mercados americano e europeu, que é de $9 \%$ e $8 \%$, respectivamente. 
A maior produtividade, peso médio dos frutos e teor de sólidos solúveis dos frutos no ambiente de Baraúnas é explicada dentre o universo de fatores, pela composição química do solo, caracterizado neste trabalho, principalmente, por ter $\mathrm{pH}(6,5)$ exigido por essa cultura. E pela qualidade da água, também relacionada ao $\mathrm{pH}(7,1)$. A eficiência da fertirrigação depende do $\mathrm{pH}$ do solo e principalmente da água, uma vez que os nutrientes são fornecidos e disponibilizados na água de irrigação; assim, o pH alto interfere negativamente na absorção de nutrientes pelas plantas (Tabelas 1 e 2).

\section{CONCLUSÕES}

Considerando-se as condições nas quais o experimento foi conduzido, pode-se concluir que não se pode recomendar um determinado híbrido de melão-amarelo para toda a região produtora de melão do Oeste potiguar, devido cada ambiente dessa região apresentar as suas peculiaridades de tipo de solo, água, luminosidade, temperatura, pluviosidade, ocorrência de pragas e doenças, dentre outras.

\section{AGRADECIMENTOS}

Ao CNPq, VALEFRUTAS, FRUNORTE, J. Pereira Produtora e Distribuidora de Frutas Ltda. e ESAM, pela alocação de recursos financeiros e apoio em infra-estrutura.

\section{REFERÊNCIASBIBLIOGRÁFICAS}

ARAÚJO, A. de P.; NEGREIROS, M. Z. de; BEZERRA NETO, F.; PEDROSA, J. F.; FERREIRA, R. L. Cobertura do solo e métodos de plantio na produção do melão. Horticultura Brasileira, São Pedro, v.18, suplemento, p.517-518, 2000.

COSTA, C. P., PINTO, C.A.B.P. Melhoramento do melão. In: Melhoramento de hortaliças. Piracicaba: USP-ESALQ, 1977. p. 161-75.

COSTA, N. D.; QUEIROZ, M. A. de; DIAS, R. de C. S.; FARIA, C. M. B de. PINTO, J. M. Desempenho de cultivares de melão no Submédio São Francisco. Horticultura Brasileira, São Pedro, v.18, suplemento, p.518-520, 2000.

COSTA, N.D.; QUEIRÓZ, M.A.; DIAS, R. de C.S.; FARIA, C.M.B.; PINTO, J.M.; RESENDE, G.M. Comportamento de cultivares de melão no Vale do São Francisco. Horticultura Brasileira, Brasília, v. 19, suplemento, julho 2001. CD-ROM.

DIAS, R. C. S.; COSTA, N. D.; CERDAN, C.; SILVA, P. C. G.; QUEIROZ M. A.; ZUZA, F.; LEITE, L. A. S.; PESSOA, P. F. A. P.; TERAO, D. A Cadeia produtiva do melão no Nordeste. In: CASTRO A. M. G.; LIMA, S. M. V.; GOEDERT, W. J.; FILHO, A. F.; VASCONCELOS, J. R. P. Cadeias produtivas e sistemas naturais: Prospeção Tecnológica.
Brasília: SPI, 1998, p. 440-493.

FERREIRA, P. V. Análise conjunta de experimentos. In: FERREIRA, P. V. Estatística experimental aplicada à agronomia. 3. ed. Maceió: EDUFAL, 2000. Cap 9.

GOMES, F.P. Os testes ou provas de significância. In: GOMES, F.P. Curso de estatística experimental. 13 ed. Piracicaba: Nobel, 1990. p.1841.

GORGATTI NETTO, A.; GAYET, J.P.; BLEINROTH, E.W. et al. Melão para exportação: procedimentos de colheita e pós-colheita. Brasília: EMBRAPA-SPI, 1994.37p. (FRUPEX. Publicações Técnicas,6).

LOPES, M.M. Caracteres descritivos e estimativas de parâmetros genéticos de cruzamento dialélico parcial entre cinco cultivares de melão (Cucumis melo L.). 1991. 45f. Dissertação (Mestrado em Fitotecnia) Escola Superior de Agricultura de Mossoró, Mossoró, 1991.

MENEZES, J.B.; GOMES, Jr. J.; ARAÚJO NETO, S. E. de; SIMÕES, A. do $\mathrm{N}$. Armazenamento de dois genótipos de melão-amarelo sob condições ambiente. Horticultura Brasileira, Jaboticabal, v. 19, n. 1, p. 4249, mar. 2001

NUNES, M. U. C.; ANDRADE, L. N. T.; ALMEIDA, A. Avaliação da produtividade, qualidade do fruto e incidência de doenças em híbridos de melão em Sergipe. Horticultura Brasileira, São Pedro, v.18, suplemento, p.553-554, 2000 .

PINHEIRO NETO, L.G.; PAIVA, W. O.; MEDEIROS, D.O.; MARQUES, R.N.; LIMA, A.F.M.; VIEIRA, F.C. Desempenho de híbridos de melão do grupo inodorus. Horticultura Brasileira, Brasília, v.19, suplemento, julho 2001. CD-ROM.

SCOTT, G.W.; MacGILLIVRAY, J.H. Variation in solids of the juice from different regions in melon fruits. Hilgardia, Oakland, v.13, n.2, 1940.

SILVA, L. A. da; INNECO, R.; COSTA, J. T. A.; MELO, F. I. O.; MALUF, W. R.; ANDRADE, C. M. G. Estudo da resistência e conteúdo de sólidos solúveis em frutos de melão. Horticultura Brasileira, Brasília, v.18, suplemento p.659-660,2000a.

SILVA, L.A. da; INNECO, R.; COSTA, J.T.A.; MELO, F.I. O.; MALUF, W. R.; ANDRADE, C. M. G. Avaliação do potencial produtivo de híbridos, cultivares e linhagens de melão para o Nordeste. Horticultura Brasileira, Brasília, v.18, suplemento, p.658-659, 2000b.

SILVA, L.A.; INNECCO, R.; ALCANFOR, D.C.; MALUF, W.R.; ANDRÉ, C.M.G. Avaliação de híbridos experimentais em relação a genótipos referenciais de melão. Horticultura Brasileira, Brasília, v. 19, suplemento 2001. CD-ROM.

TORRES FILHO, J.; BEZERRA NETO, F.; HOLANDA, J.S. et al. Adaptabilidade ambiental e estabilidade produtiva de 15 cultivares de caupi na Serra do Mel. Pesquisa Agropecuária Brasileira, Brasília, v.22, n.6, p.485-490, 1987. 